archives-ouvertes

\title{
Third-order Complex Amplitudes Tracking Loop for Slow Fading Channel Estimation
}

\author{
Huaqiang Shu, Laurent Ros, Eric Simon
}

\section{To cite this version:}

Huaqiang Shu, Laurent Ros, Eric Simon. Third-order Complex Amplitudes Tracking Loop for Slow Fading Channel Estimation. ICT 2012 - 19th International Conference on Telecommunications, Apr 2012, Jounieh, Lebanon. 6 p. hal-00701523

\section{HAL Id: hal-00701523 \\ https://hal.archives-ouvertes.fr/hal-00701523}

Submitted on 25 May 2012

HAL is a multi-disciplinary open access archive for the deposit and dissemination of scientific research documents, whether they are published or not. The documents may come from teaching and research institutions in France or abroad, or from public or private research centers.
L'archive ouverte pluridisciplinaire HAL, est destinée au dépôt et à la diffusion de documents scientifiques de niveau recherche, publiés ou non, émanant des établissements d'enseignement et de recherche français ou étrangers, des laboratoires publics ou privés. 


\section{Third-order Complex Amplitudes Tracking Loop for Slow Fading Channel Estimation}

\author{
Huaqiang SHU \\ IEMN lab, TELICE group \\ 59655 Villeneuve d'Ascq \\ University of Lille, France
}

\author{
Laurent ROS \\ GIPSA-Lab, Image and Signal Department \\ BP 46, 38402 Saint Martin d'Hères \\ France
}

\author{
Eric Pierre SIMON \\ IEMN lab, TELICE group \\ 59655 Villeneuve d'Ascq \\ University of Lille, France
}

\begin{abstract}
This paper deals with channel estimation over a flat fading Rayleigh channel with Jakes' Doppler Spectrum. Many estimation algorithms exploit the time-domain correlation of the channel by employing a Kalman filter based on a firstorder (or sometimes second-order) approximation of the timevarying channel. In the low-variation channel scenario, generally speaking, a well-chosen higher order estimator can perform better than a lower order one (Ros et al., [1] [2]). Based on this fact, we propose a third-order tracking loop estimator inspired by the principle of the phase-locked loop (PLL). The proposed estimator has a less complex structure compared to the Kalmanbased estimators. In addition, the mean-squared-error (MSE) of the proposed estimator is studied, as well as the parameter optimization with the aim of minimizing the MSE. The closed form expression of the optimal MSE is given and validates the interest of our approach.
\end{abstract}

Index Terms-Channel estimation, Rayleigh fading, Jakes' spectrum, Phase-locked loop (PLL), Kalman filter (KF).

\section{INTRODUCTION}

Channel estimation is a fundamental task for a wireless communication receiver. This paper deals with channel path Complex Amplitude (CA) estimators. Many channel path CA tracking algorithms use a Kalman Filter (KF) based on a linear recursive approximation model of the widely accepted Rayleigh fading channel with Jakes' Doppler spectrum ( [2][8]). However, KF based algorithms exhibit a certain complexity, without ensuring optimal performance if the approximation model is not well suited, or if the model coefficients are slightly out of tune, as we will develop hereafter. A widely used channel approximation model results from a first-order Auto-Regressive model (AR1) as recommended by [9], combined with a Correlation Matching (CM) criterion to fix the AR1-coefficient (equal then to the standard Bessel AR1-coefficient, $J_{0}\left(2 \pi f_{d} T\right)$, for a given normalized Doppler frequency $f_{d} T$ ). The $\mathrm{KF}$ channel estimator resulting from this choice, called $\mathrm{AR} 1_{C M}$-KF in this paper, was used in various systems such as in Multiple-Input-Multiple-Output systems [3], [4], or in Orthogonal Frequency Division Multiplexing (OFDM) systems [5], [6], [10], [11].

The AR $1_{C M}$-KF seems to be convenient for the very high mobility case, leading to quasi-optimal channel estimation performance compared to lower bounds, as seen, for example, in [10]-[12] (in these works the $\mathrm{AR} 1_{C M}-\mathrm{KF}$ is actually used to track the Basis Extension Model coefficients of the high speed channel). But for most conventional Doppler speeds such as the channel variation within one symbol duration can be neglected (i.e. $f_{d} T \leq 10^{-2}$, as in [1]-[8]), the MSE performance of the widely used $\mathrm{AR} 1_{C M}-\mathrm{KF}$ estimator is somewhat disappointing, as we have recently pointed out in [1] (see Fig.2). It was observed that the asymptotic MSE performance of the $\mathrm{AR} 1_{C M}$ - KF is largely poorer than that of less complex algorithms in [1] based on simple secondorder Complex Amplitude Tracking Loops (CATL) inspired by Phase-Locked Loops (PLL), and is, above all, very far from the Bayesian Cramer Rao lower Bound (BCRB) ( [13]). This poor performance has just been explained analytically in [8], mainly because the $\mathrm{CM}$ criterion is shown to be inappropriate to tune the AR1-coefficient in slowly fading scenario (since the choice of $J_{0}\left(2 \pi f_{d} T\right) \approx 1-\frac{1}{4}\left(2 \pi f_{d} T\right)^{2}$ for the AR1coefficient is too close to the value 1 to ensure a good tradeoff between tracking ability and noise mitigation). A better tuning of the AR1-coefficient can focus on minimizing the estimation variance in output of the $\mathrm{KF}$ as proposed in [7] (with analytic MSE performance for a given Doppler and SNR scenario in [8]), i.e. using a minimum asymptotic variance (MAV) criterion without imposing the CM constraint. The resulting KF is called here AR $1_{M A V}-\mathrm{KF}$. On the other hand, [2] shows analytically that, the MSE performance of a KF can still be improved by switching from the AR1 model to an integrated random walk model for the approximation model (called here Or2-KF), exploiting the strong trend behaviour of the CA variation, since the CA continues in some direction during several symbols for low $f_{d} T$.

To sum up, second-order channel tracking algorithms seem to be more appropriate than first-order algorithms for slow fading scenario, in the KF version ( [2]) as well as in the CATL version ( [1]), but without reaching the lower bound BCRB. The multi-fold contributions of this paper is to give positive answers to the questions that arise: can a well-chosen thirdorder CATL have the ability to outperform the asymptotic MSE performance of the more complex KF based only on first- or second-order models (i.e. $\mathrm{AR} 1_{C M}-\mathrm{KF}, \mathrm{AR} 1_{M A V}-\mathrm{KF}$, or Or2-KF)? How to tune properly and in a simple way the coefficients of such a third-order CATL, assuming RayleighJakes channel and a given scenario of $f_{d} T$ and SNR? What is then the closed form expression of the MSE of such a channel estimator? 
Section II gives the system model. In section III, we propose and analyze a third-order Complex-Amplitude-Tracking Loop, called Or3-CATL, for the estimation of the slowly timevarying channel. Section IV describes a proposed method to correctly tune the loop coefficients, and section $\mathrm{V}$ validates our model and assumptions by means of simulations.

\section{Model and Estimation ObJective}

We consider the estimation of a flat Rayleigh fading channel. The discrete-time observation is ${ }^{1}$ :

$$
y_{(n)}=\alpha_{(n)}+N_{(n)}
$$

where $n$ is the symbol time index, $N_{(n)}$ is a zero-mean additive white circular complex Gaussian noise with variance $\sigma_{N}^{2}$, and $\alpha_{(n)}$ is a zero-mean circular Gaussian channel Complex Amplitude with variance $\sigma_{\alpha}^{2}=1$. The normalized Doppler frequency of this channel is $f_{d} T$, where $T$ is the symbol period. A Jakes' Doppler spectrum is assumed for this channel:

$$
\Gamma_{\alpha}(f)=\left\{\begin{array}{ccc}
\frac{\sigma_{\alpha}^{2}}{\pi f_{d} \sqrt{1-\left(\frac{f}{f_{d}}\right)^{2}}}, & \text { if } & |f|<f_{d} \\
0, & \text { if } & |f| \geq f_{d} .
\end{array}\right.
$$

The autocorrelation matrix $R_{\alpha}[m]$ of the stationary CA $\alpha$ is then defined for lag $m$ by:

$$
R_{\alpha}[m]=E\left\{\alpha_{(n)} \cdot \alpha_{(n-m)}{ }^{*}\right\}=\sigma_{\alpha}^{2} J_{0}\left(2 \pi f_{d} T \cdot m\right)
$$

where $J_{0}$ is the zeroth-order Bessel function of the first kind. Given the observation model (1) and the Doppler spectrum statistical constraint (2) for the dynamic evolution of the CA, we look for an on-line unbiased estimation $\hat{\alpha}_{(n)}$ of $\alpha_{(n)}$. The variance $\sigma_{\epsilon}^{2} \stackrel{\text { def }}{=} E\left\{\left|\epsilon_{(n)}\right|^{2}\right\}$ of the estimation error $\epsilon_{(n)} \stackrel{\text { def }}{=}$ $\alpha_{(n)}-\hat{\alpha}_{(n)}$ will be investigated.

\section{Complex Amplitude Tracking LoOP}

\section{A. Structure}

Inspired first by the principle of a PLL [14] (intended for the phase estimation), we propose for our CA estimation a thirdorder PLL-like structure, called CATL (Complex Amplitude Tracking Loop). Similarly to a classical digital PLL, the CATL is composed of an error detector, a loop filter and a numerically controlled generator. The error detector compares firstly the received signal with a reference signal equal to the previous prediction of the parameter, $\hat{\alpha}_{(n \mid n-1)}$. It delivers the error signal $v_{\epsilon(n)}$ to the Lead-Lag filter (or Proportional-Integral filter) which is controlled by three parameters $\mu_{1}, \mu_{2}$ and $\mu_{3}$. The output $v_{c(n)}$ is then used by the numerically controlled generator to generate the next prediction of parameter $\hat{\alpha}_{(n+1 \mid n)}=\hat{\alpha}_{(n \mid n-1)}+v_{c(n)}$. The structure is shown in Fig.1. It is similar to a third-order PLL [15]. However, unlike the conventional PLL, the final output of the CATL is not directly the prediction $\hat{\alpha}_{(n \mid n-1)}$ but the final estimate of the complex

\footnotetext{
${ }^{1}$ Model (1) assumes that symbols are normalized and known (or decided), additionally to flat fading assumption. Although this model is admittedly simplistic, it can be applied to different (more involved) contexts, such as pilot-aided multi-carrier systems in frequency-selective wireless channels.
}

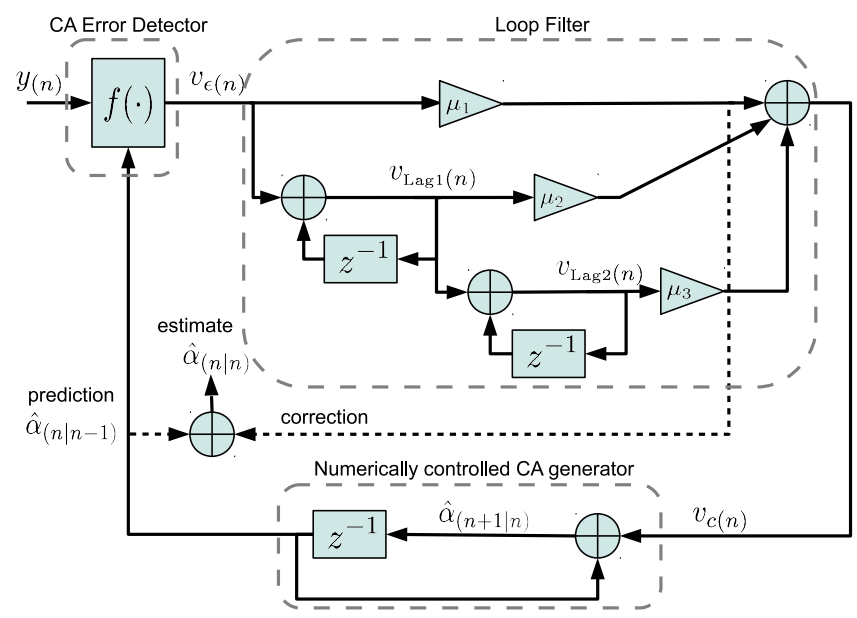

Fig. 1: Equivalent structure of the Or3-CATL, inspired by the third-order digital PLL

amplitude $\hat{\alpha}_{(n \mid n)}$, as in the KF principle. Thus an additional branch of correction is required. As for a KF [16], the CATL can be described by two-stage recursive equations:

Measurement Update Equations

$$
\begin{aligned}
v_{\epsilon(n)} & =y_{(n)}-\hat{\alpha}_{(n \mid n-1)} \\
\hat{\alpha}_{(n \mid n)} & =\hat{\alpha}_{(n \mid n-1)}+\mu_{1} v_{\epsilon(n)}
\end{aligned}
$$

Time Update Equations

$$
\begin{aligned}
v_{\text {Lag1 } 1}(n) & =v_{\text {Lag1 }(n-1)}+v_{\epsilon(n)} \\
v_{\text {Lag2 }(n)} & =v_{\text {Lag2 }(n-1)}+v_{\text {Lag1 } 1 n)} \\
\hat{\alpha}_{(n+1 \mid n)} & =\hat{\alpha}_{(n \mid n)}+\mu_{2} v_{\text {Lag1 } 1(n)}+\mu_{3} v_{\text {Lag2 }(n)}
\end{aligned}
$$

where $\mu_{1}, \mu_{2}, \mu_{3}$ are the filter coefficients (real positive).

The Measurement Update Equations are responsible for the feedback, i.e., for incorporating a new measurement $y_{(n)}$ into the a priori estimate $\hat{\alpha}_{(n \mid n-1)}$ to obtain an improved $a$ posteriori estimate $\hat{\alpha}_{(n \mid n)}$. The Time Update Equations are responsible for projecting forward in time the current state $\hat{\alpha}_{(n \mid n)}$ to obtain the next a priori estimate $\hat{\alpha}_{(n+1 \mid n)}$, by adding a component proportional to $v_{\mathrm{Lag} 1(n)}$ and $v_{\mathrm{Lag} 2}(n)$. The signals $v_{\text {Lag1 }(n)}, v_{\operatorname{Lag} 2(n)}$ defined in (6) and (7) are respectively the firstorder and the second-order digital integrations (or accumulations) of the error signal $v_{\epsilon(n)}$. Note that, thanks to the second integration in $v_{\text {Lag2 }(n)}$, this digital third-order loop does not exhibit acceleration-dependent steady-state error in the case of second-order variations of the CAs. In other words, the Or3CATL characterizes the variation of the channel parameter by taking into account its slope and its curvature, while secondorder loops only consider the slope.

\section{B. General properties and theoretical MSE analysis}

1) Third-order CATL close loop transfer function: by combining (1) and (4), we have :

$$
v_{\epsilon(n)}=\left(\alpha_{(n)}-\hat{\alpha}_{(n \mid n-1)}\right)+N_{(n)}
$$


so that the error signal is a combination of the prediction error $\left(\alpha_{(n)}-\hat{\alpha}_{(n \mid n-1)}\right)$ and the channel noise. By combining (5) and (9), we obtain the error signal - estimation error relation:

$$
v_{\epsilon(n)}=\frac{1}{1-\mu_{1}} \cdot\left(\alpha_{(n)}-\hat{\alpha}_{(n \mid n)}\right)+\frac{1}{1-\mu_{1}} \cdot N_{(n)}
$$

Then by combining (5) and (8), we obtain the estimation update equation:

$$
\hat{\alpha}_{(n \mid n)}=\hat{\alpha}_{(n-1 \mid n-1)}+\mu_{1} v_{\epsilon(n)}+\mu_{2} v_{\mathrm{Lag} 1(n-1)}+\mu_{3} v_{\mathrm{Lag} 2(n-1)}
$$

And with (6) and (7), the Z-domain expression of (11) is obtained:

$$
\hat{\alpha}(z)\left[1-z^{-1}\right]=\left[\mu_{1}+\frac{\mu_{2} \cdot z^{-1}}{1-z^{-1}}+\frac{\mu_{3} \cdot z^{-1}}{\left(1-z^{-1}\right)^{2}}\right] \cdot v_{\epsilon}(z)
$$

Then using (9) leads to

$$
\hat{\alpha}(z)=L(z) \cdot \alpha(z)+L(z) \cdot N(z)
$$

where $L(z)$ is the Z-domain transfer function of the 3rd-order CATL defined by (14) with $F(z)=\mu_{1}+\frac{\mu_{2} \cdot z^{-1}}{1-z^{-1}}+\frac{\mu_{3} \cdot z^{-1}}{\left(1-z^{-1}\right)^{2}}$ the transfer function of the loop filter. It can be rewritten in a more interpretable form as a function of the natural pulsation $\omega_{n}$, the damping factor $\zeta$ and the capacitance ratio $m$ as (15), where:

$$
\begin{aligned}
m \zeta \cdot\left(\omega_{n} T\right)^{3} & =\frac{\mu_{3}}{1-\mu_{1}} \\
\left(1+2 m \zeta^{2}\right) \cdot\left(\omega_{n} T\right)^{2} & =\frac{\mu_{2}-\mu_{3}}{1-\mu_{1}} \\
(m+2) \cdot \zeta \omega_{n} T & =\frac{\mu_{1}-\mu_{2}}{1-\mu_{1}}
\end{aligned}
$$

The capacitance ratio $m$ is an additional factor for thirdorder PLL used to adjust the step response character [17]. By comparing (14) and (15), $\left(\mu_{1}, \mu_{2}, \mu_{3}\right)$ can be expressed by $\left(\omega_{n}, \zeta, m\right)$ as:

$$
\begin{aligned}
& \mu_{1}=\frac{(m+2) \zeta \omega_{n} T+\left(1+2 m \zeta^{2}\right)\left(\omega_{n} T\right)^{2}+m \zeta\left(\omega_{n} T\right)^{3}}{1+(m+2) \zeta \omega_{n} T+\left(1+2 m \zeta^{2}\right)\left(\omega_{n} T\right)^{2}+m \zeta\left(\omega_{n} T\right)^{3}} \\
& \mu_{2}=\frac{\left(1+2 m \zeta^{2}\right)\left(\omega_{n} T\right)^{2}+m \zeta\left(\omega_{n} T\right)^{3}}{1+(m+2) \zeta \omega_{n} T+\left(1+2 m \zeta^{2}\right)\left(\omega_{n} T\right)^{2}+m \zeta\left(\omega_{n} T\right)^{3}} \\
& \mu_{3}=\frac{m \zeta\left(\omega_{n} T\right)^{3}}{1+(m+2) \zeta \omega_{n} T+\left(1+2 m \zeta^{2}\right)\left(\omega_{n} T\right)^{2}+m \zeta\left(\omega_{n} T\right)^{3}}
\end{aligned}
$$

If we impose the constraint that $\omega_{n}>0, \zeta>0$ and $m>0$ to preserve a physical meaning, we deduce from (16), (17) and (18) that $0<\mu_{3}<\mu_{2}<\mu_{1}<1$. We can rewrite $L(z)$ in the frequency-domain, by making $z=e^{p T}$, with $p=j \omega=j 2 \pi f$. Assuming slow reaction of the loop during one symbol time $T$ (i.e. $f_{n} T \ll 1$ ), the digital loop transfer function is close (approximation $z^{-1} \approx 1-p T$ ) to

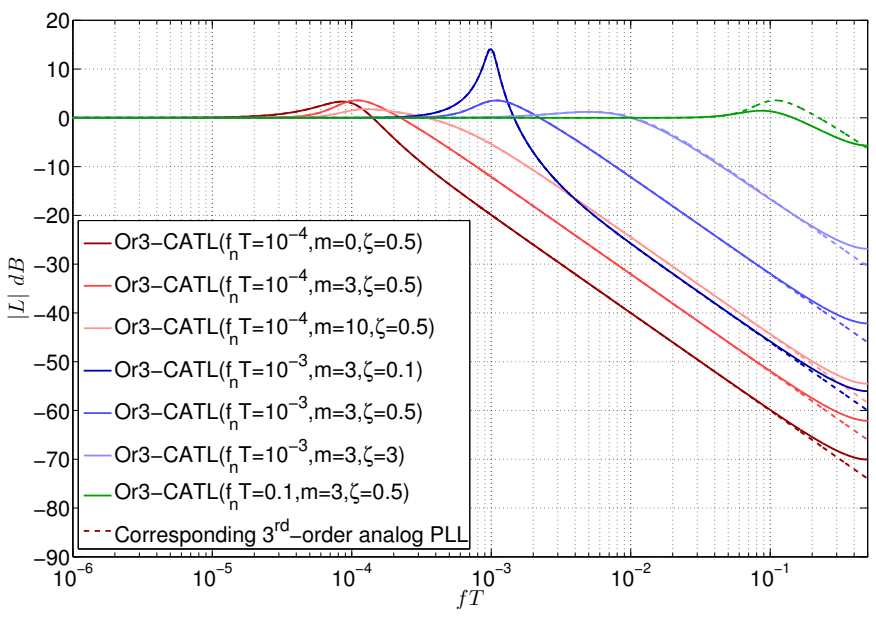

Fig. 2: Comparison of Transfer functions $L$ of the the Or3CATL (15) (continuous curves) and of 3rd-order analog PLL (22) (dashed curves) with different set $\left(m, \zeta, f_{n}\right)$ versus $f T$

the usual third-order low-pass transfer function in analog PLL:

$$
L\left(e^{p T}\right) \approx \frac{(m+2) \zeta \omega_{n} \cdot p^{2}+\left(1+2 m \zeta^{2}\right) \omega_{n}^{2} \cdot p+m \zeta \omega_{n}^{3}}{p^{3}+(m+2) \zeta \omega_{n} \cdot p^{2}+\left(1+2 m \zeta^{2}\right) \omega_{n}^{2} \cdot p+m \zeta \omega_{n}^{3}}
$$

Fig. 2 shows the magnitude-frequency graph of the digital third-order CATL transfer function and the analog third-order PLL transfer function, respectively given by (15) and (22) with different natural frequencies. We can see that in the low-frequency domain $\left(f_{n} T \ll 1\right)$, the two transfer functions match very well, and then the analog version gives a good approximation of the true CATL transfer function.

2) Mean squared error analysis: from (13) we know that the estimation error is zero-mean, thus the third-order CATL is an unbiased estimator. By using the definition $\epsilon(z)=\alpha(z)-$ $\hat{\alpha}(z),(13)$ can be re-written as:

$$
\epsilon(z)=(1-L(z)) \cdot \alpha(z)-L(z) \cdot N(z)
$$

The variance of estimation error is therefore divided into two parts. One comes from the variation of the parameter $\alpha$ and the other comes from the loop noise $N$ :

$$
\sigma_{\epsilon}^{2}=E\left\{\epsilon_{(n)} \cdot \epsilon_{(n)}^{*}\right\}=\sigma_{\epsilon \alpha}^{2}+\sigma_{\epsilon N}^{2}
$$

The component $\sigma_{\epsilon \alpha}^{2}$ (dynamic error variance) results from the high-pass filtering $(1-L(z))$ of the input CAs $\alpha_{(n)}$, so we

$$
\begin{gathered}
L(z)=\frac{F(z)}{\left(1-\mu_{1}\right)\left(1-z^{-1}\right)+F(z)}=\frac{\left[\left(\mu_{1}-\mu_{2}\right)\left(1-z^{-1}\right)^{2}+\left(\mu_{2}-\mu_{3}\right)\left(1-z^{-1}\right)+\mu_{3}\right]}{\left(1-\mu_{1}\right)\left(1-z^{-1}\right)^{3}+\left[\left(\mu_{1}-\mu_{2}\right)\left(1-z^{-1}\right)^{2}+\left(\mu_{2}-\mu_{3}\right)\left(1-z^{-1}\right)+\mu_{3}\right]} \\
L(z)=\frac{(m+2) \zeta \omega_{n} \cdot\left(1-z^{-1}\right)^{2}+\left(1+2 m \zeta^{2}\right) \omega_{n}^{2} \cdot\left(1-z^{-1}\right)+m \zeta \omega_{n}^{3}}{\left(1-z^{-1}\right)^{3}+(m+2) \zeta \omega_{n} \cdot\left(1-z^{-1}\right)^{2}+\left(1+2 m \zeta^{2}\right) \omega_{n}^{2} \cdot\left(1-z^{-1}\right)+m \zeta \omega_{n}^{3}}
\end{gathered}
$$


have

$$
\sigma_{\epsilon \alpha}^{2}=\int_{-\frac{1}{2 T}}^{+\frac{1}{2 T}} \Gamma_{\alpha}(f) \cdot\left|1-L\left(e^{j 2 \pi f T}\right)\right|^{2} d f
$$

where $\Gamma_{\alpha}(f)$ is the PSD of $\alpha$ given by (2). And the component $\sigma_{\epsilon N}^{2}$ (static error variance) results from the low-pass filtering $L(z)$ of the input loop noise $N_{(n)}$ :

$$
\sigma_{\epsilon N}^{2}=\int_{-\frac{1}{2 T}}^{+\frac{1}{2 T}} \Gamma_{N}(f) \cdot\left|L\left(e^{j 2 \pi f T}\right)\right|^{2} d f
$$

a) Static error variance $\sigma_{\epsilon N}^{2}$ : since the noise is assumed white, the PSD of noise $\Gamma_{N}(f)=\sigma_{N}^{2} T$ is constant all over the system bandwidth. Thus (26) can be re-written by

$$
\sigma_{\epsilon N}^{2}=\sigma_{N}^{2} \cdot \underbrace{T \int_{-\frac{1}{2 T}}^{+\frac{1}{2 T}}\left|L\left(e^{j 2 \pi f T}\right)\right|^{2} d f}_{B_{L}}
$$

where $B_{L}$ is the so-called equivalent noise bandwidth (doublesided normalized). $B_{L}$ can be derived (i.e. to evaluate the two-sided complex integral) by using the method presented in [18]. For a third-order system, $B_{L}$ is a sixth-degree algebraic expression of $\omega_{n} T$. But with the condition $f_{n} T \ll 1$ in our case, the high-order terms of $\omega_{n} T$ are negligible, so that $B_{L}$ can be finally approximated as:

$$
B_{L} \approx 2 \pi f_{n} T \cdot \underbrace{\frac{\left(2 m^{3} \zeta^{4}+12 m^{2} \zeta^{4}+8 m \zeta^{4}+6 m \zeta^{2}+4 \zeta^{2}+1\right)}{4 m^{2} \zeta^{3}+8 m \zeta^{3}+4 \zeta}}_{\mathscr{B}}
$$

b) Dynamic error variance $\sigma_{\epsilon \alpha}^{2}$ : for the Rayleigh-Jakes model, the Doppler spectrum has a bounded support $f \in]-$ $f_{d},+f_{d}[$ (see (2)). Therefore, a good tracking of $\alpha$ requires that the natural frequency of the Or3-CATL $f_{n}$ be greater than the Doppler frequency $f_{d}$. On the other hand, assuming $f_{n} \ll$ $1 / T$, we can use the approximation (22) which yields:

$$
\begin{aligned}
& \left.\left|1-L\left(e^{j 2 \pi f T}\right)\right|^{2} \quad \text { (for }|f| \leq f_{d}\right)
\end{aligned}
$$

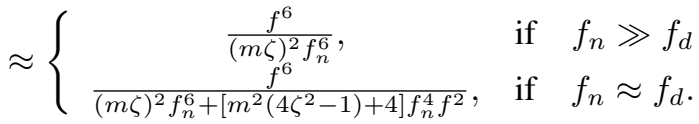

Indeed, if $f_{n} \gg f_{d}$, then $f_{n} \gg f$ since $f<f_{d}$. Hence, the denominator can be simplified to $(m \zeta)^{2} f_{n}^{6}$. If $f_{n} \approx f_{d}$, the previous simplification does not hold any more, yielding a second term (see (29)). In order to be able to still neglect this second term in the perspective to obtain an analytical formula of the integral (25), we suggest choosing $(m, \zeta)$ such that:

$$
m^{2}\left(4 \zeta^{2}-1\right)+4=0
$$

The consequences of such a constraint will be discussed later. With this constraint, $\left|1-L\left(e^{j 2 \pi f T}\right)\right|^{2}$ can be considered as a linear function of $\left(\frac{f}{f_{n}}\right)^{6}$ for $|f| \leq f_{d}$ (for any $f_{n}$ such that $\left.f_{d} \leq f_{n} \ll 1 / T\right)$, and thus the dynamic error variance $\sigma_{\epsilon \alpha}^{2}$ becomes:

$$
\sigma_{\epsilon \alpha}^{2} \approx \frac{1}{(m \zeta)^{2}} \int_{-f_{d}}^{+f_{d}} \Gamma_{\alpha}(f) \cdot\left(\frac{f}{f_{n}}\right)^{6} \cdot d f
$$

For the Rayleigh-Jakes model, a variable change $\cos (\theta)=\left(f / f_{d}\right)$ permits us to compute an exact analytical solution of the integral (31) as:

$$
\sigma_{\epsilon \alpha}^{2} \approx \frac{5}{16} \cdot \frac{1}{(m \zeta)^{2}} \cdot\left(\frac{f_{d}}{f_{n}}\right)^{6} \cdot \sigma_{\alpha}^{2}
$$

\section{COMPUTATION OF THE OR3-CATL PARAMETERS}

The MSE $\sigma_{\epsilon}^{2}(24)$ is minimized for an optimal parameters set $\left(m, \zeta, f_{n}\right)$ obtained through a three dimensional optimization. A closed-form analytical expression for this problem can be obtained if we impose the constraint (30), leading to a suboptimal solution. We present in this section a method to obtain the sub-optimal parameters, and we show that the result is very close to the optimal solution.

\section{A. Computation of $(m, \zeta)$}

Let $\mathscr{C}$ be the set of couples $(m, \zeta)$ which satisfy the constraint (30). There is an infinite number of couples in $\mathscr{C}$ and the one that will be selected minimizes the MSE. Fig. 3 shows the MSE as a function of $(m, \zeta)$ for SNR $=0 \mathrm{~dB}$ and $f_{d} T=10^{-3}$. The MSE (24) is obtained by numerical integration of (25) and (26). For each MSE value of the figure, the best $f_{n} T$ has been selected (such that the MSE is minimum) by carrying out a numerical optimization. It is noteworthy that there exists a "valley-belt" in which the minimum MSE is located. The set $\mathscr{C}$ has also been plotted (dashed line), yielding a second-degree curve in the $m-\zeta$ plane. The sub-optimal parameters $(m, \zeta)$ are those which belong to both $\mathscr{C}$ and the bottom of the valley (triangle point in Fig. 3). A more intuitive 3D view of the MSE valley is illustrated in Fig. 4. The results given by numerical minimization are as follows: for $\mathrm{SNR}=0 \mathrm{~dB}, m=3.196, \zeta=0.39$; for $\mathrm{SNR}=20 \mathrm{~dB}, m=2.974, \zeta=0.37$ and for $\mathrm{SNR}=40$ $\mathrm{dB}, m=3.078, \zeta=0.38$. We can see that, for different SNRs, the sub-optimal $m$ is always around 3 and does not vary strongly. Hence, $m$ is fixed to 3 . Then from (30), we get $\zeta=\frac{\sqrt{5}}{6}$. To sum up, we have selected a sub-optimal couple $(m, \zeta)$ satisfying (30) with a corresponding MSE very close to the global minimum MSE. It remains for us now to provide an analytical formula for $f_{n}$.

\section{B. Optimal natural frequency $f_{n}$}

Now that the couple $(m, \zeta)$ is set, we can calculate the natural frequency $f_{n}$ (for $f_{d}<f_{n} \ll 1 / T$ ) that permits us to minimize the MSE $\sigma_{\epsilon}^{2}$ in (24) (assuming (28) and (32)) as:

$$
\left(\frac{f_{n}}{f_{d}}\right)(\text { Jakes })=\left[\frac{15}{16} \cdot \frac{1}{\pi \mathscr{B}} \cdot \frac{1}{(m \zeta)^{2}} \cdot \frac{1}{f_{d} T} \cdot \frac{\sigma_{\alpha}^{2}}{\sigma_{N}^{2}}\right]^{\frac{1}{7}}
$$

yielding the sub-optimal MSE:

$$
\sigma_{\epsilon}^{2}(\text { Jakes })=\lambda \cdot\left(\sigma_{\alpha}^{2}\right)^{\frac{1}{7}} \cdot\left(\sigma_{N}^{2} \cdot f_{d} T\right)^{\frac{6}{7}}
$$

where:

$$
\lambda=\frac{35}{16} \cdot\left(\mathscr{B} \cdot \frac{16 \pi}{15}\right)^{\frac{6}{7}} \cdot\left[\frac{1}{(m \zeta)^{2}}\right]^{\frac{1}{7}}
$$

with $\mathscr{B}$ defined in (28). Note that the above equations hold for any $(m, \zeta)$ satisfying the constraint $(30)$. 


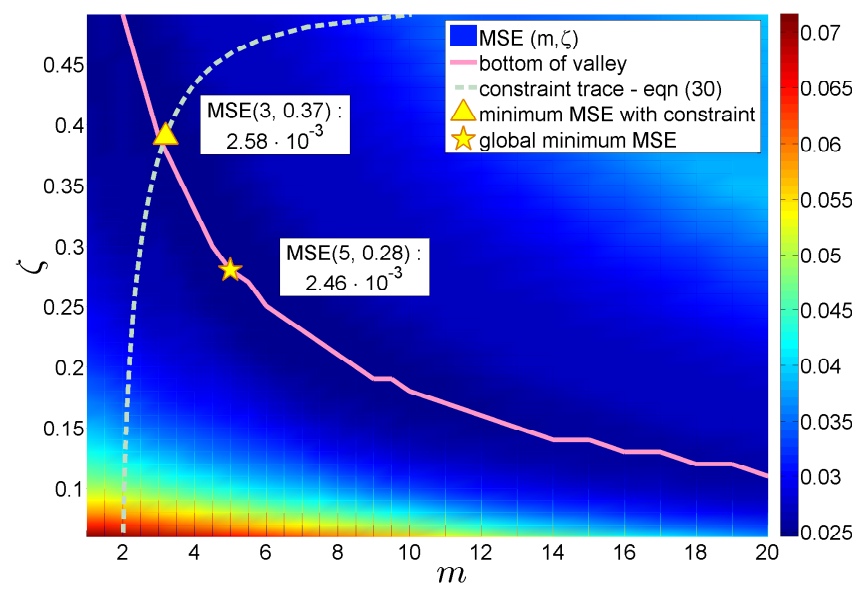

Fig. 3: MSE (24) versus $(m, \zeta)$ computed by numerical integration of (25) and (26), SNR $=0 \mathrm{~dB}$

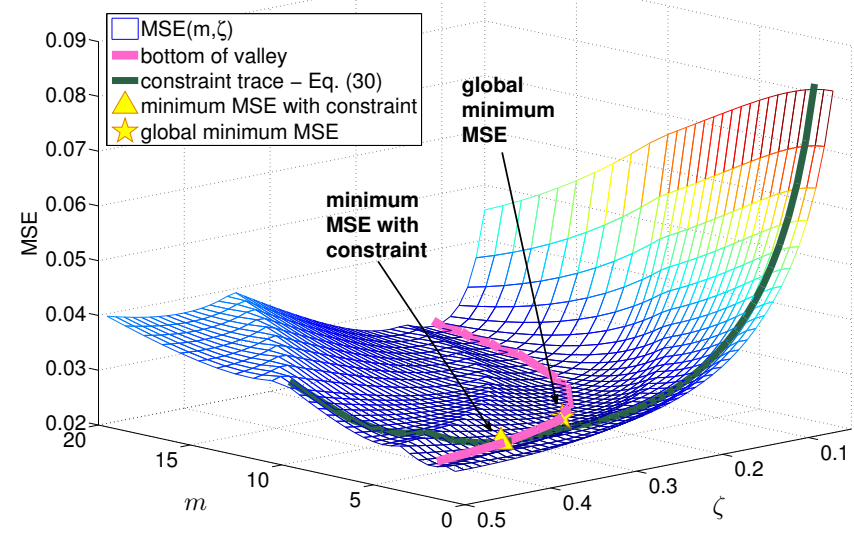

Fig. 4: Another view of the MSE (24) versus $(m, \zeta)$ computed by numerical integration of (25) and (26), SNR $=0 \mathrm{~dB}$

\section{Simulation}

In this section, the performance of the Or3-CATL in terms of MSE is assessed through simulations, and is compared to that of reference algorithms based on Kalman. For all our simulations the channel autocorrelation function is assumed to be given by the widely accepted Jakes' model, as stated in Section II.

Fig. 5 gives the comparison between simulated and theoretical MSE versus $f_{n}$ for $f_{d} T=10^{-3}$, and $\mathrm{SNR}=0,20$ and $40 \mathrm{~dB}$. The sub-optimal loop parameters $\left(m=3, \zeta=\frac{\sqrt{5}}{6}\right)$ are considered (see section IV-A). The theoretical dynamic and static error variances (dashed lines) $\sigma_{\epsilon \alpha}^{2}$ and $\sigma_{\epsilon N}^{2}$ are obtained by numerical integration of (25) and (26), respectively. The approximated error variances (square points) computed by the approximated formulae ((27) with (28) and (32)) are also plotted. It is observed that the approximated MSEs match very well the theoretical MSEs. On the other hand, we can also observe that the component $\sigma_{\epsilon \alpha}^{2}$ is the main contribution of $\sigma_{\epsilon}^{2}$ for small $f_{n}$, whereas the component $\sigma_{\epsilon N}^{2}$ dominates when $f_{n}$ increases. This is understood from (32) and (28) since $\sigma_{\epsilon \alpha}^{2}$

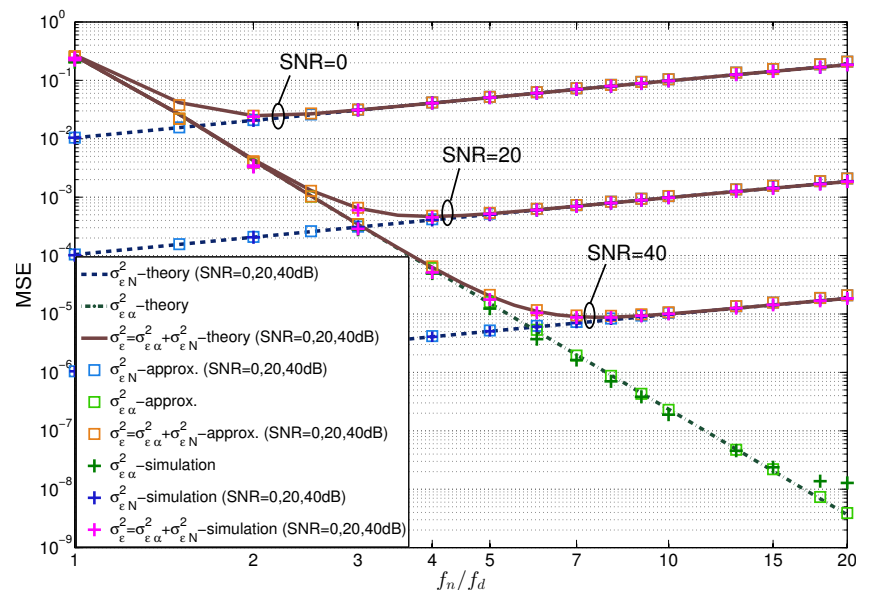

Fig. 5: Theoretical and simulated MSEs vs $f_{n} / f_{d}$ (Or3-CATL with $m=3, \zeta=\frac{\sqrt{5}}{6}, \mathrm{SNR}=0,20,40 \mathrm{~dB}, f_{d} T=10^{-3}$ )

is inversely proportional to $f_{n}^{6}$, while $\sigma_{\epsilon N}^{2}$ is proportional to $f_{n}$. Simulated MSEs have also been plotted. The simulated dynamic error variance $\sigma_{\epsilon \alpha}^{2}$ was obtained by forcing the noise $N_{(n)}$ to zero, whereas the simulated static error variance $\sigma_{\epsilon N}^{2}$ was obtained by maintaining the CA to a constant value equal to its standard deviation $\sigma_{\alpha}$. First of all, we can observe that all the theoretical curves are very close to the simulated ones, which validates our theoretical analysis and our approximations. Therefore, the abscissa of the minimum of the simulated MSE $\sigma_{\epsilon}^{2}$ matches also very well with the (theoretical closed form (33)) optimal natural frequency (such that $f_{n} / f_{d}$ (Jakes) $=2,3.9$ and 7.6 respectively for SNR = 0,20 , and $40 \mathrm{~dB}$ ).

Fig. 6 compares the MSE of the Or3-CATL with that of the $\mathrm{AR} 1_{C M}-\mathrm{KF}$ [3]-[6], the $\mathrm{AR} 1_{M A V}-\mathrm{KF}$ [7] [8] and the Or2KF [2] by means of Monte-Carlo simulations for $f_{d} T=10^{-4}$ and $f_{d} T=10^{-3}$. Note that our proposed Or3-CATL algorithm assumes the same a priori knowledge as that required for the KF (Jakes model, noise variance, Doppler frequency). We also plot the on-line BCRB as reference [13]. It is observed that the MSE performance of the $\mathrm{AR} 1_{C M}-\mathrm{KF}$ is very poor. This result corroborates the works cited in the introduction, which point out that the $\mathrm{AR} 1_{C M}-\mathrm{KF}$ is convenient for high mobility $\left(f_{d} T>>10^{-2}\right)$, but exhibits poor performance at $f_{d} T \leq$ $10^{-2}$ as proved by [8]. As expected, the Or2-KF performs better than $\mathrm{AR} 1_{C M}-\mathrm{KF}$ and $\mathrm{AR} 1_{M A V}-\mathrm{KF}$. Finally, the MSE of the Or3-CATL with the loop parameters properly chosen (see section IV) is the closest to the BCRB (which could be concluded from the MSE expressions of the 4 estimators). This result shows that it is preferable to use a well-chosen thirdorder algorithm based on simple CATL to a Kalman Filter when the later is based only on first- or second-order models.

Fig. 7 shows the MSE of the different systems versus $f_{d} T$. The gain in performance of the Or3-CATL is greater for small values of $f_{d} T$. When $f_{d} T$ increases, the MSEs of the $\mathrm{AR} 1_{M A V}-\mathrm{KF}$, Or2-KF and Or3-CATL systems seem to converge to the MSE of the $\mathrm{AR} 1_{C M}-\mathrm{KF}$. 


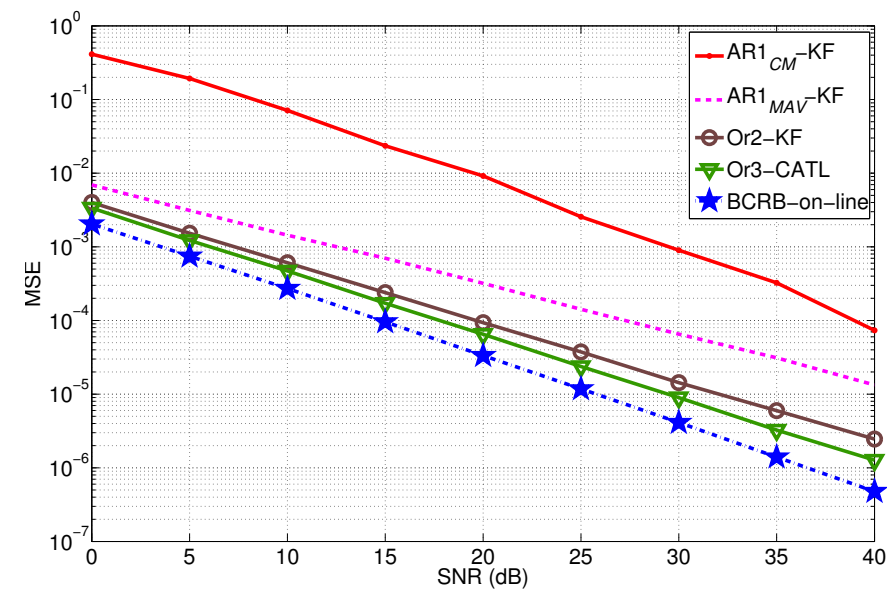

(a) $f_{d} T=10^{-4}$

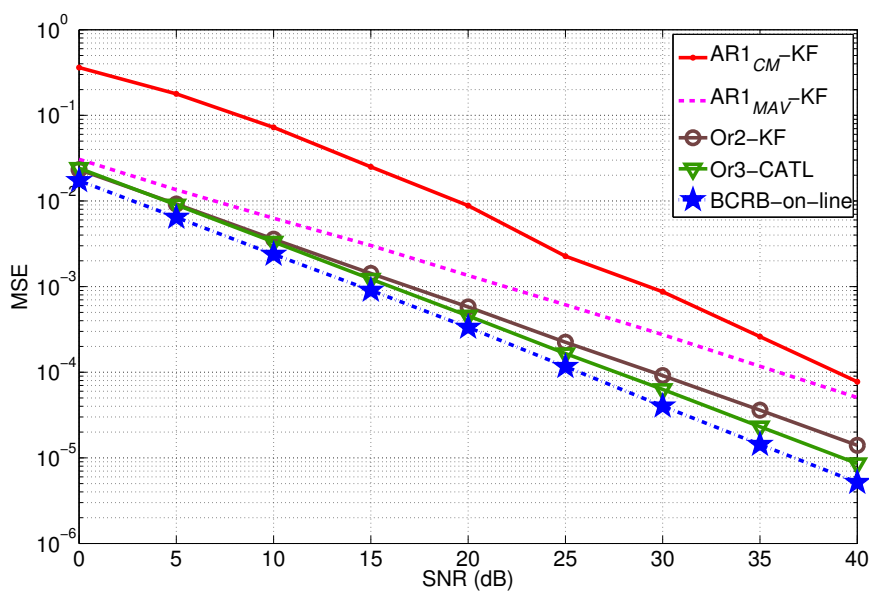

(b) $f_{d} T=10^{-3}$

Fig. 6: MSE of the Or3-CATL compared to literature versus SNR for (a) $f_{d} T=10^{-4}$ (b) $f_{d} T=10^{-3}$

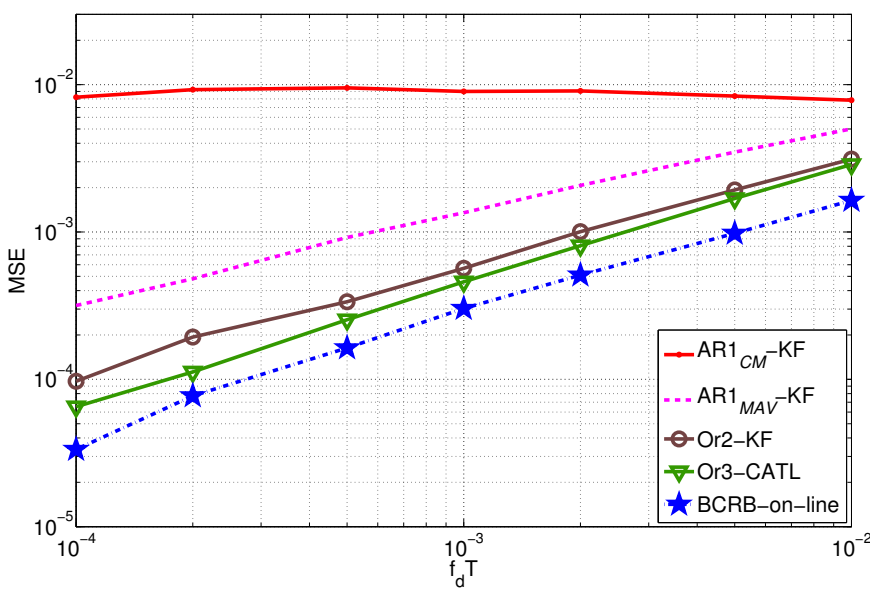

Fig. 7: MSE versus $f_{d} T, \mathrm{SNR}=20 \mathrm{~dB}$

\section{CONCLUSION}

In this paper, a channel path complex amplitude estimator over slow fading channels has been proposed and analyzed. The simple case of a flat fading channel was considered, but the results can be applied or generalized to more complex systems, such as wireless OFDM systems. Our proposed estimator is based on a third-order tracking loop. We have also proposed a simple method to properly tune the three loop parameters. We have demonstrated that, by fixing the capacitance ratio to 3 , the damping factor to $\frac{\sqrt{5}}{6}$, and by computing the natural frequency with a given expression depending on the Doppler frequency, it is possible to achieve near-optimal performance in terms of MSE. Simulation results show that, with these well-chosen parameters, the proposed algorithm outperforms the more complex Kalman filter based algorithms of the literature (based on first- or second-order models), as long as the mobility is moderate (i.e. $f_{d} T<10^{-2}$ ), which is a very common scenario.

\section{REFERENCES}

[1] L. Ros, H. Hijazi, and E. P. Simon, "Paths complex gain tracking algorithms for OFDM receiver in slowly-varying channels," in Proc. IEEE ISCCSP, Mar 2010, pp. 1 - 6.

[2] L. Ros and E. P. Simon, "Second-order modeling for Rayleigh flat fading channel estimation with Kalman Filter," in Proc. IEEE DSP, Jul 2011, pp. $1-6$.

[3] C. Komninakis, C. Fragouli, A. H. Sayed, and R. D. Wesel, "Multi-input multi-output fading channel tracking and equalization using Kalman estimation," IEEE Trans. Signal Process., vol. 50, no. 5, pp. 1065 1076, May 2002.

[4] Z. Liu, X. Ma, and G. Giannakis, "Space-time coding and Kalman filtering for time-selective fading channels," IEEE Trans. Commun., vol. 50, no. 2, pp. 183 - 186, Feb 2002.

[5] W. Chen and R. Zhang, "Kalman-filter channel estimator for OFDM systems in time and frequency-selective fading environment," in Proc. IEEE ICASSP, vol. 4, May 2004, pp. iv-377 - iv-380.

[6] T. Y. Al-Naffouri, "An EM-Based Forward-Backward Kalman Filter for the Estimation of Time-Variant Channels in OFDM," IEEE Trans. Signal Process., vol. 55, no. 7, pp. 3924 - 3930, Jul 2007.

[7] A. Barbieri, A. Piemontese, and G. Colavolpe, "On the ARMA approximation for fading channels described by the Clarke model with applications to Kalman-based receivers," IEEE Trans. Wireless Commun., vol. 8, no. 2, pp. 535 - 540, Feb 2009.

[8] S. Ghandour-Haidar, L. Ros, and J. M. Brossier, "On the Use of first-order Autoregressive Modeling for Rayleigh Flat Fading Channel Estimation with Kalman filter," ELSEVIER Signal Proc., vol. 92, no. 2, pp. $601-606$, Feb 2012.

[9] H. S. Wang and P.-C. Chang, "On verifying the first-order Markovian assumption for a Rayleigh fading channel model," IEEE Trans. Veh. Technol., vol. 45, no. 2, pp. 353 - 357, May 1996.

[10] H. Hijazi and L. Ros, "Joint data QR-detection and Kalman estimation for OFDM time-varying Rayleigh channel complex gains," IEEE Trans. Commun., vol. 58, no. 1, pp. 170 - 178, Jan 2010.

[11] E. P. Simon, L. Ros, H. Hijazi, and M. Ghogho, "Joint Carrier Frequency Offset and Channel Estimation for OFDM Systems via the EM Algorithm in the presence of very high mobility," in IEEE Trans. Signal Process., accepted to be published in 2012.

[12] J. K. Tugnait, S. He, and H. Kim, "Doubly Selective Channel Estimation Using Exponential Basis Models and Subblock Tracking," IEEE Trans. Signal Process., vol. 58, no. 3, pp. 1275 - 1289, Mar 2010.

[13] H. Hijazi and L. Ros, "Bayesian Cramer-Rao Bounds for OFDM Slowly Varying Multi-path Rayleigh Channel Estimation," in ELSEVIER Signal Proc. Fast Communication, vol. 89, Jan 2009, pp. 111 -115.

[14] U. Mengali and A. N. D'Andrea, Synchronization Techniques for Digital Receivers. Plenum Press, 1997.

[15] W. C. Lindsey and C. M. Chie, "A survey of digital phase-locked loops," Proc. IEEE, vol. 69, no. 4, pp. 410 - 431, Apr 1981.

[16] S. M. Kay, Fundamentals of Statistical Signal Processing : Estimation Theory. Prentice Hall PTR, Apr 5, 1993.

[17] S. Hu, Principle of Automatic Control (4th edition). Beijing: Science Press, 2001.

[18] R. Winkelstein, "Closed form evaluation of symmetric two-sided complex integrals," TDA Progress Report, pp. 42 - 65, Jul \& Aug, 1981. 